\title{
WZW models as mutual super Poisson-Lie T-dual sigma models
}

\author{
Department of Physics, Faculty of science, Azarbaijan Shahid Madani University, \\ 53714-161, Tabriz, Iran \\ E-mail: a.eghbali@azaruniv.edu, rezaei-a@azaruniv.edu
}

\begin{abstract}
A WZW model on the Lie supergroup $\left(C^{3}+A\right)$ is constructed. It is shown that this model contains super Poisson-Lie symmetry with the dual Lie supergroup $C^{3} \oplus A_{1,1} . i$. Furthermore, we show that the dual model is also equivalent to the WZW model on isomorphic Lie supergroup $\left(C^{3}+A\right) . i$. In this manner, because of isomorphism of the $\left(\mathcal{C}^{3}+\mathcal{A}\right)$ with a Manin supertriple, it is shown that the $\mathrm{N}=(2,2)$ structure is preserved under the super Poisson-Lie T-duality transformation.
\end{abstract}

KeYwords: Conformal and W Symmetry, String Duality, Sigma Models. 


\section{Contents}

1. Introduction 1

2. A review of super Poisson-Lie T-duality 2

3. Super Poisson-Lie symmetry in the $\left(C^{3}+A\right)$ WZW model

4. The (2|2)-dimensional super Poisson-Lie T-dual sigma models on the $\left(\left(C^{3}+A\right), C^{3} \oplus A_{1,1} \cdot i\right)$

5. Preservation of the $\mathrm{N}=(2,2)$ structure under super Poisson-Lie Tduality 9

6. Conclusion and remarks 10

\section{Introduction}

The WZW models on Lie supergroups have recently received considerable attention, because of their relations to local logarithmic conformal field theories [1]-[3]. Superstring theory on $A d S$ backgrounds relates to WZW model on the Lie supergroups and the coset superspaces [4]-[7]. On the other hand, duality symmetries play an important role in string theory. They are specific to string theory and their study led to important insights in understanding the geometry of space-time from the string point of view. A very important symmetry of string theories, or more generally, two-dimensional sigma models is the T-duality 8]. It has been shown that the conformal symmetry is preserved under the Abelian duality [9], and also this duality has been investigated in the WZW model (see for example [10]). Furthermore for the non-Abelian duality case [11], it has been shown that the conformal symmetry is preserved when the trace of the adjoint representation of the isometry group is zero [12]. The Poisson-Lie T-duality [13] is a generalization of Abelian and non-Abelian target space duality (T-duality). This generalized duality is associated with the two groups forming a Drinfel'd double such that the duality transformation exchanges their roles. Up to now, there is one example for the conformal Poisson-Lie T-dual sigma models [14]; such that the duality relates the $S L(2, R)$ WZW model to a constrained sigma model. Recently the generalization of the Poisson-Lie T-duality in sigma models on supermanifolds has been performed in [15, 16]. Furthermore, we have shown that the WZW model on the Lie supergroup $G L(1 \mid 1)$ has also super Poisson-Lie symmetry when the dual Lie supergroup is $B \oplus A \oplus A_{1,1} . i$ [17]. Here we first show that the WZW model on the Lie supergroup $\left(C^{3}+A\right)$ contains super Poisson-Lie symmetry, then we describe a pair of super 
Poisson-Lie T-dual sigma models which is associated with the $\left(\left(C^{3}+A\right), C^{3} \oplus A_{1,1} \cdot i\right)$ Drinfel'd superdouble such that the original model is the WZW model on the $\left(C^{3}+A\right)$; moreover, we show that the dual model is also equivalent to the WZW model on isomorphic Lie supergroup $\left(C^{3}+A\right) . i$. Thus, in this way we have found an example in which conformal invariance is preserved under the super Poisson-Lie T-duality transformation. In fact, this is the first example of the conformal sigma models related by super Poisson-Lie T-duality which has so far been constructed. Furthermore, because of isomorphism of the $\left(\mathcal{C}^{3}+\mathcal{A}\right)$ with a Manin supertriple we show that the $\mathrm{N}=(2,2)$ structure is preserved under the super Poisson-Lie T-duality transformation for this example.

The structure of the paper is as follows. In Section 2, for introducing of the notations and self-containing of the subject, we briefly review some aspects of the super Poisson-Lie symmetry and T-dual sigma model on Lie supergroup [15]. In Section 3, by using the condition of the super Poisson-Lie symmetry we show that the WZW model on $\left(C^{3}+A\right)$ Lie supergroup contains super Poisson-Lie symmetry when the dual Lie supergroup is $C^{3} \oplus A_{1,1} . i$. Then, we obtain the mutually T-dual sigma models on the Drinfel'd superdouble $\left(\left(C^{3}+A\right), C^{3} \oplus A_{1,1} \cdot i\right)$ in Section 4 such that the original model is the same as the $\left(C^{3}+A\right)$ WZW model. The proof of the conformal invariance for the dual model is also given in Section 4. Furthermore, we show that T-dual sigma models have flat backgrounds. Indeed, the dual model is equivalent to the WZW model on isomorphic Lie supergroup $\left(C^{3}+A\right) . i$. Finally, in Section 5 by use of the fact that the algebraic representation of the $\mathrm{N}=(2,2)$ structure is a Manin triple and the $\left(\mathcal{C}^{3}+\mathcal{A}\right)$ Lie superalgebra is a Manin supertriple; we show that the $\mathrm{N}=(2,2)$ structure is preserved under the super Poisson-Lie T-duality transformation.

\section{A review of super Poisson-Lie T-duality}

Consider a nonlinear sigma model described by a supersymmetric metric $G_{\Upsilon \Lambda}$ and a super antisymmetric two-form field $B_{\Upsilon \Lambda}$ on the target supermanifold $M$ (the superdimension of the supermanifold is written as (\# bosons $\mid$ \# fermions) $=\left(d_{B} \mid d_{F}\right)$, which, because of the invertibility of the metric $G_{\Upsilon \Lambda}, d_{F}$ must be even [18]) with the action ${ }^{1}$

$$
\begin{aligned}
S & =\frac{1}{2} \int d \xi^{+} d \xi^{-}(-1)^{|\Upsilon|} \partial_{+} \Phi^{\Upsilon} \mathcal{E}_{\Upsilon \Lambda} \partial_{-} \Phi^{\Lambda} \\
& =\frac{1}{2} \int d \xi^{+} d \xi^{-}(-1)^{|\Upsilon|} \partial_{+} \Phi^{\Upsilon}\left(G_{\Upsilon \Lambda}+B_{\Upsilon \Lambda}\right) \partial_{-} \Phi^{\Lambda},
\end{aligned}
$$

where $\xi^{ \pm} \equiv \frac{1}{2}(\tau \pm \sigma)$ are the standard light-cone variables on the worldsheet and $\Phi^{\Upsilon}$ are coordinates on $M$, which include the bosonic coordinates $X^{\mu}\left(\mu=0, \cdots, d_{B}-1\right)$ and the fermionic ones $\Theta^{\alpha}\left(\alpha=1, \cdots, d_{F}\right)$, and the labels $\Upsilon$ and $\Lambda$ run over $(\mu, \alpha)$. Let now

$$
\star J_{i}=(-1)^{\Lambda+\Upsilon \Lambda} V_{i}^{\Lambda} \mathcal{E}_{\Upsilon \Lambda} \partial_{+} \Phi^{\Upsilon} d \xi^{+}-(-1)^{\Lambda} V_{i}^{\Lambda} \mathcal{E}_{\Lambda \Upsilon} \partial_{-} \Phi^{\Upsilon} d \xi^{-},
$$

\footnotetext{
${ }^{1}$ We note that the $(-1)^{|\Upsilon|}$ denotes the parity of $\Upsilon$ where $|\Upsilon|=0$ for the bosonic coordinates and $|\Upsilon|=1$ for the fermionic coordinates. Here and in the following we use the notation presented by DeWitt [1] ], e.g., $(-1)^{\Upsilon}:=(-1)^{|\Upsilon|}$.
} 
be the Hodge star of Noether's current one-forms corresponding to the right action of the Lie supergroup $G$ on the target $M$, in which $V_{i}^{\Upsilon}(\Phi)$ 's are the left invariant supervector fields defined with left derivative [15]. If the forms $\star J_{i}$ not be closed and on the extremal surfaces satisfy the Maurer-Cartan equation [18], we say that the sigma model has the super Poisson-Lie symmetry with respect to the Lie supergroup $\tilde{G}$ (the dual Lie supergroup to $G)$ [15. It is a condition on $\mathcal{E}_{\Upsilon \Lambda}$ so that it can be formulated as 15

$$
\mathcal{L}_{V_{i}}\left(\mathcal{E}_{\Upsilon \Lambda}\right)=(-1)^{i(\Upsilon+k)} \mathcal{E}_{\Upsilon \Xi}\left(V^{s t}\right)_{k}^{\Xi}\left(\tilde{\mathcal{Y}}_{i}\right)^{k j}{ }_{j} V^{\Omega}{ }_{\Omega} \mathcal{E}_{\Lambda},
$$

where $\left(\tilde{\mathcal{Y}}_{i}\right)^{j k}=-\tilde{f}^{j k}{ }_{i}$ are the adjoint representations of Lie superalgebra $\tilde{\mathcal{G}}$ (the dual Lie superalgebra to $\mathcal{G}$ ) and "st" stands for the supertranspose [18].

Let us suppose now that $G$ acts transitively and freely on $M$; then the mutually $T$-dual sigma models have target spaces in the Lie supergroups $G$ and $\tilde{G}$ and are defined by the actions $^{2}$

$$
\begin{aligned}
S & =\frac{1}{2} \int d \xi^{+} d \xi^{-}(-1)^{i} R_{+}^{(l)^{i}} E_{i j}^{+}(g) R_{-}^{(l)^{j}}, & & g \in G, \\
\tilde{S} & =\frac{1}{2} \int d \xi^{+} d \xi^{-}(-1)^{j} \tilde{R}_{+i}^{(l)} \tilde{E}^{+i j}(\tilde{g}) \tilde{R}_{-j}^{(l)}, & & \tilde{g} \in \tilde{G},
\end{aligned}
$$

where

$$
R^{(l)^{i}}=\left(d g g^{-1}\right)^{i}=(-1)^{\Upsilon} d \Phi^{\Upsilon} R_{\Upsilon}^{(l)}{ }^{i}
$$

and

$$
\tilde{R}_{i}^{(l)}=\left(d \tilde{g} \tilde{g}^{-1}\right)_{i}=(-1)^{\Upsilon} d \tilde{\Phi}^{\Upsilon} \tilde{R}^{(l)} \Upsilon_{i},
$$

are right invariant one-forms (defined with left derivative). Here the matrices $E(g)$ and $\tilde{E}(\tilde{g})$ are defined in the following way ${ }^{3}$

$$
E^{+}(g)=\left(\Pi(g)+\left(E_{0}^{+}\right)^{-1}(e)\right)^{-1}, \quad \tilde{E^{+}}(\tilde{g})=\left(\tilde{\Pi}(\tilde{g})+\left(\tilde{E}_{0}^{+}\right)^{-1}(\tilde{e})\right)^{-1},
$$

in which $E_{0}^{+}(e)$ and $\tilde{E}_{0}^{+}(\tilde{e})$ are constant matrices such that in the vicinity of the origin of the Lie supergroup $(g=e$ and $\tilde{g}=\tilde{e})$ are related to each other as

$$
{ }_{i} E_{0_{j}}^{+}(e) \tilde{E}_{0}^{+j k}(\tilde{e})={ }_{i} \delta^{k},
$$

and $\Pi^{i j}(g)=(-1)^{k} b^{i k}(g)\left(a^{-1}\right)_{k}^{j}(g)$, where the matrices $a(g)$ and $b(g)$ are constructed in the following way

$$
\begin{aligned}
g^{-1} X_{i} g & =(-1)^{j} a_{i}{ }^{j}(g) X_{j}, \\
g^{-1} \tilde{X}^{i} g & =(-1)^{j} b^{i j}(g) X_{j}+d^{i}{ }_{j}(g) \tilde{X}^{j} .
\end{aligned}
$$

Here $X_{i}$ and $\tilde{X}^{i}$ are basis of the respective Lie superalgebras $\mathcal{G}$ and $\tilde{\mathcal{G}}$ where form a pair of maximally superisotropic Lie subsuperalgebras into the Drinfel'd superdouble ${ }^{4}$ so that

$$
<X_{i}, X_{j}>=<\tilde{X}^{i}, \tilde{X}^{j}>=0,
$$

\footnotetext{
${ }^{2}$ The construction of the models has been described in 15 .

${ }^{3}$ Here, one must use of superdeterminant and superinverse formulas 18 .

${ }^{4}$ A Drinfel'd superdouble [19] is a Lie superalgebra $\mathcal{D}$ which decomposes into the direct sum, as supervector spaces, of two maximally superisotropic Lie subsuperalgebras $\mathcal{G}$ and $\tilde{\mathcal{G}}$, each corresponding to a Poisson-Lie supergroup $(G$ and $\tilde{G})$, such that the Lie subsuperalgebras are duals of each other in the usual sense, i.e., $\tilde{\mathcal{G}}=\mathcal{G}^{*}$.
} 


$$
<X_{i}, \tilde{X}^{j}>=(-1)^{i j}<\tilde{X}^{j}, X_{i}>=(-1)^{i}{ }_{i} \delta^{j}
$$

and their structure constants satisfy in the following mixed super Jacobi identities 22]:

$$
f_{j k}^{m} \tilde{f}_{m}^{i l}=f_{m k}^{i} \tilde{f}_{j}^{m l}+f_{j m}^{l} \tilde{f}_{k}^{i m}+(-1)^{j l} f_{j m}^{i} \tilde{f}_{k}^{m l}+(-1)^{i k} f_{m k}^{l} \tilde{f}_{j}^{i m} .
$$

\section{Super Poisson-Lie symmetry in the $\left(C^{3}+A\right)$ WZW model}

In what follows we shall construct a WZW model based on the Lie supergroup $\left(C^{3}+\right.$ $A$ ) of dimension- $(2 \mid 2)$. Indeed in [19] it has been shown that this Lie supergroup is the same as the Drinfel'd superdouble $D=\left(\left(A_{1,1}+A\right), I_{(1 \mid 1)}\right)$ in such a way that its Lie superalgebra $\left(\mathcal{C}^{3}+\mathcal{A}\right)$ is a (1|1)-dimensional Lie superbialgebra [20] and has the following explicit description:

$$
\left[H, Q_{-}\right]=Q_{+}, \quad\left[H, Q_{+}\right]=0, \quad\left\{Q_{-}, Q_{-}\right\}=Z, \quad\left\{Q_{+}, Q_{ \pm}\right\}=0, \quad[Z, .]=0,
$$

where $H, Z$ and $Q_{+}, Q_{-}$are bosonic generators and fermionic ones, respectively. To define a WZW model, one needs a bilinear form $\Omega_{i j}$ in the generators $X_{i}$, which is supersymmetric $\left(\Omega_{i j}=(-1)^{i j} \Omega_{j i}\right)$ ad-invariant metric on $\mathcal{G}$ and satisfies in the following relation

$$
f_{i j}^{l} \Omega_{l k}+(-1)^{j k} f_{i k}^{l} \Omega_{l j}=0 .
$$

A non-degenerate solution to (3.2) exists and is given by

$$
\Omega_{i j}=\left(\begin{array}{cccc}
b & a & 0 & c \\
a & 0 & 0 & d \\
0 & 0 & 0 & -a \\
c & d & a & 0
\end{array}\right)
$$

where $a, b, c$ and $d$ are real constants and $a$ is non-zero. Then the WZW action on a Lie supergroup $G$ is given by 17

$$
\begin{aligned}
S_{W Z W}(g)=\frac{k}{4 \pi} & \int_{\Sigma} d^{2} \xi(-1)^{i} L_{+}^{(l) i} \Omega_{i j} L_{-}^{(l) j} \\
& \quad-\frac{k}{24 \pi} \int_{B} d^{3} \xi(-1)^{i+j k} \varepsilon^{\gamma \alpha \beta} L_{\gamma}^{(l) i} \Omega_{i l} L_{\alpha}^{(l) j}\left(\mathcal{Y}^{l}\right)_{j k} L_{\beta}^{(l) k},
\end{aligned}
$$

where the $L_{\alpha}^{(l) i}$ 's are defined via $g^{-1} \partial_{\alpha} g=(-1)^{i} L_{\alpha}^{(l) i} X_{i}$ (with $g: \Sigma \rightarrow G$ ) and $B$ is a three-manifold bounded by worldsheet $\Sigma$. To write explicit form of the action (3.4) on $G$ we choose a general element of the Lie supergroup $\left(C^{3}+A\right)$ as

$$
g=e^{\chi Q_{-}} e^{y H+x Z} e^{\psi Q_{+}}
$$

where $x(\tau, \sigma)$ and $y(\tau, \sigma)$ are bosonic fields while $\psi(\tau, \sigma)$ and $\chi(\tau, \sigma)$ are fermoinic ones. Then $L_{\alpha}^{(l) i}$ 's are found to be

$$
L_{\alpha}^{(l) H}=\partial_{\alpha} y,
$$




$$
\begin{aligned}
L_{\alpha}^{(l) Z} & =\partial_{\alpha} x-\partial_{\alpha} \chi \frac{\chi}{2}, \\
L_{\alpha}^{(l) Q_{+}} & =-\partial_{\alpha} \psi+\partial_{\alpha} \chi y, \\
L_{\alpha}^{(l) Q_{-}} & =-\partial_{\alpha} \chi .
\end{aligned}
$$

Furthermore, the terms that are being integrated over in (3.4) are calculated to be

$$
\begin{gathered}
L_{+}^{(l) i}{ }_{i} \Omega_{j} L_{-}^{(l) j}=a\left[\partial_{+} y \partial_{-} x+\partial_{+} x \partial_{-} y-\partial_{+} \psi \partial_{-} \chi+\partial_{+} \chi \partial_{-} \psi\right. \\
\left.+\partial_{+} y \frac{\chi}{2} \partial_{-} \chi-\partial_{+} \chi \frac{\chi}{2} \partial_{-} y\right] \\
(-1)^{j k} L_{\gamma}^{(l) i}{ }_{i} \Omega_{l} L_{\alpha}^{(l) j}\left(\mathcal{Y}^{l}\right)_{j k} L_{\beta}^{(l) k}=a \partial_{\gamma}\left[y \partial_{\alpha} \chi \partial_{\beta} \chi-\chi \partial_{\alpha} y \partial_{\beta} \chi+\chi \partial_{\alpha} \chi \partial_{\beta} y\right] .
\end{gathered}
$$

Hence, the WZW action on the Lie supergroup $\left(C^{3}+A\right)$ is written as

$$
S_{W Z W}(g)=\frac{1}{2} \int_{\Sigma} d \xi^{+} d \xi^{-}\left(\partial_{+} y \partial_{-} x+\partial_{+} x \partial_{-} y-\partial_{+} \psi \partial_{-} \chi+\partial_{+} \chi \partial_{-} \psi+\partial_{+} y \chi \partial_{-} \chi\right) .
$$

Here we have assumed that $b=c=d=0$ in (3.3), and $a$ has rescaled to $\frac{2 \pi}{k}$. By identifying the action (3.9) with the sigma model of the form (2.1), one can read off the background matrix as

$$
\mathcal{E}_{\Upsilon \Lambda}=\left(\begin{array}{cccc}
0 & 1 & 0 & \chi \\
1 & 0 & 0 & 0 \\
0 & 0 & 0 & 1 \\
0 & 0 & -1 & 0
\end{array}\right)
$$

As showned in the above, the background matrix is a function of the fermionic field $\chi$ only. In order to find super Poisson-Lie symmetry in WZW model on the Lie supergroup $\left(C^{3}+A\right)$, we need the left invariant supervector fields (defined with left derivative) on the $\left(C^{3}+A\right)$. By applying relations (3.6) in the relation ${ }_{i} V^{\Upsilon}=\left(\Upsilon L^{i}\right)^{-1}$ [15 the supervector fields are calculated to be

$$
\begin{aligned}
{ }_{H} V & =\frac{\vec{\partial}}{\partial y} \\
{ }_{z} V & =\frac{\vec{\partial}}{\partial x} \\
{ }_{Q_{+}} V & =-\frac{\vec{\partial}}{\partial \psi}, \\
{ }_{Q_{-}} V & =-\frac{\chi}{2} \frac{\vec{\partial}}{\partial x}-y \frac{\vec{\partial}}{\partial \psi}-\frac{\vec{\partial}}{\partial \chi} .
\end{aligned}
$$

Now by calculation of the Lie superderivatie of the bi-tensor $\mathcal{E}_{\Upsilon \Lambda}$ (3.10) with respect to ${ }_{i} V^{\prime}$ s and then by substituting relations (3.10) and (3.11) on the right hand side of Eq. (2.3), 
after some calculations the non-zero structure constants of the dual pair to the $\left(\mathcal{C}^{3}+\mathcal{A}\right)$ Lie superalgebra are found to be

$$
\left[\tilde{Z}, \tilde{Q}_{+}\right]=-\frac{1}{2} \tilde{Q}_{-}
$$

On the other hand we have been recently classified all (2|2)-dimensional decomposable Lie superalgebras 21. The Lie superalgebra obtained in (3.12) is isomorphic to the decomposable trivial Lie superalgebra $\mathcal{C}^{3} \oplus \mathcal{A}_{1,1}$ [21] under the following transformation:

$$
\begin{aligned}
\tilde{H} & =a Z^{\prime}, \\
\tilde{Z} & =b H^{\prime}+c Z^{\prime}, \\
\tilde{Q}_{+} & =-d Q^{\prime}{ }_{+} e Q^{\prime}{ }_{-}, \\
\tilde{Q}_{-} & =2 b e Q^{\prime}{ }_{+},
\end{aligned}
$$

where $a, b, e \in \Re-\{0\} ; c, d \in \Re$. The generetors $\left\{H^{\prime}, Z^{\prime}\right\}$ and $\left\{Q_{+}^{\prime}, Q_{-}^{\prime}\right\}$ are bosonic and fermionic generators of the Lie superalgebra $\mathcal{C}^{3} \oplus \mathcal{A}_{1,1}$, respectively. Hence we denote the dual pair to the $\left(\mathcal{C}^{3}+\mathcal{A}\right)$ by $\mathcal{C}^{3} \oplus \mathcal{A}_{1,1} . i$ with the same of the commutation relation obtained in (3.12). Two sets of generators (3.1) and (3.12) are dual to each other in the sense of 2.11). Nevertheless, one can check that the Lie superbialgebra $\left(\left(\mathcal{C}^{3}+\mathcal{A}\right), \mathcal{C}^{3} \oplus \mathcal{A}_{1,1}, i\right)$ satisfies mixed super Jacobi identities (2.12) [22]. The fact that the WZW model on the Lie supergroup $\left(C^{3}+A\right)$ has super Poisson-Lie symmetry is quite interesting. In the following, we shall construct the mutually T-dual sigma models on the Drinfel'd superdouble $\left(\left(C^{3}+A\right), C^{3} \oplus A_{1,1} . i\right)$ and will show that the original sigma model on the $\left(C^{3}+A\right)$ is identical to WZW model on the $\left(C^{3}+A\right)$. Moreover, to prove the conformal invariance of the dual model we will look at the one-loop $\beta$-function equations and then will discuss that it is conformal invariant for all loops.

\section{The (2|2)-dimensional super Poisson-Lie T-dual sigma models on the $\left(\left(C^{3}+A\right), C^{3} \oplus A_{1,1} \cdot i\right)$}

To construct the mutually T-dual sigma models we need the Derinfel'd superdouble which is simply a Lie supergroup $D$. The decomposition of the superdouble into the pair of maximally isotropic Lie subsuperalgebras $\mathcal{D}=\mathcal{G} \oplus \tilde{\mathcal{G}}$ is referred to as the Manin supertriple $(\mathcal{D}, \mathcal{G}, \tilde{\mathcal{G}})$. Consider the Lie superalgebra $\mathcal{G}=\left(\mathcal{C}^{3}+\mathcal{A}\right)$ defined by (anti)commutation relations (3.1) and its dual $\tilde{\mathcal{G}}=\mathcal{C}^{3} \oplus \mathcal{A}_{1,1} . i$ which is generated by relation (3.12). The Lie superalgebra of the Derinfel'd superdouble which we refer to as the $\left(\left(\mathcal{C}^{3}+\mathcal{A}\right), \mathcal{C}^{3} \oplus \mathcal{A}_{1,1} \cdot i\right)$ is denoted by generators $\left\{H, Z, \tilde{H}, \tilde{Z} ; Q_{+}, Q_{-}, \tilde{Q}_{+}, \tilde{Q}_{-}\right\}$and the following non-zero (anti) commutation relations

$$
\left[H, Q_{-}\right]=Q_{+}, \quad\left\{Q_{-}, Q_{-}\right\}=Z, \quad\left[\tilde{Z}, \tilde{Q}_{+}\right]=-\frac{1}{2} \tilde{Q}_{-},
$$




$$
\left[H, \tilde{Q}_{+}\right]=-\tilde{Q}_{-}, \quad\left[Q_{-}, \tilde{Z}\right]=-\frac{1}{2} Q_{+}-\tilde{Q}_{-}, \quad\left\{Q_{-}, \tilde{Q}_{+}\right\}=-\frac{1}{2} Z-\tilde{H} .
$$

In order to construct the corresponding original sigma model with the Lie supergroup $\left(C^{3}+A\right)$ as the target space, we use the same parametrization (3.5). Inserting this parametrization in (2.6) we find

$$
\partial_{ \pm} g g^{-1}=\partial_{ \pm} y H+\left(\partial_{ \pm} x+\partial_{ \pm} \chi \frac{\chi}{2}\right) Z+\left(\partial_{ \pm} \psi-\partial_{ \pm} y \chi\right) Q_{+}+\partial_{ \pm} \chi Q_{-}
$$

from which we can obtain $R_{ \pm}^{(l)^{i}}$ 's as

$$
\begin{aligned}
R_{ \pm}^{(l)}{ }^{H} & =\partial_{ \pm} y \\
R_{ \pm}^{(l)}{ }^{Z} & =\partial_{ \pm} x+\partial_{ \pm} \chi \frac{\chi}{2} \\
R_{ \pm}^{(l)}{ }^{Q_{+}} & =\partial_{ \pm} y \chi-\partial_{ \pm} \psi \\
R_{ \pm}^{(l)}{ }^{Q_{-}} & =-\partial_{ \pm} \chi
\end{aligned}
$$

Then the matrices $a(g), b(g)$ and $d(g)$ from Eq. (2.10) read

$$
\begin{aligned}
a_{i}^{j}(g) & =\left(\begin{array}{cccc}
1 & 0 & -\chi & 0 \\
0 & 1 & 0 & 0 \\
0 & 0 & -1 & 0 \\
0 & -\chi & y & -1
\end{array}\right), b^{i j}(g)=\left(\begin{array}{cccc}
0 & 0 & 0 & 0 \\
0 & 0 & -\frac{\chi}{2} & 0 \\
0 & \frac{\chi}{2} & 0 & 0 \\
0 & 0 & 0 & 0
\end{array}\right) \\
d^{i}{ }_{j}(g) & =\left(\begin{array}{cccc}
1 & 0 & 0 & 0 \\
0 & 1 & 0 & \chi \\
\chi & 0 & 1 & y \\
0 & 0 & 0 & 1
\end{array}\right) .
\end{aligned}
$$

Here we choose the sigma model matrix $E_{0}^{+}(e)$ at the unit element of $\left(C^{3}+A\right)$ as

$$
E_{0_{i j}}^{+}(e)=\left(\begin{array}{cccc}
0 & 1 & 0 & 0 \\
1 & 0 & 0 & 0 \\
0 & 0 & 0 & 1 \\
0 & 0 & -1 & 0
\end{array}\right)
$$

By a direct application of the first formula of (2.8), the corresponding sigma model with the Lie supergroup $\left(C^{3}+A\right)$ is worked out as follows:

$$
S=\frac{1}{2} \int d \xi^{+} d \xi^{-}\left(\partial_{+} y \partial_{-} x+\partial_{+} x \partial_{-} y-\partial_{+} \psi \partial_{-} \chi+\partial_{+} \chi \partial_{-} \psi+\partial_{+} y \chi \partial_{-} \chi\right) .
$$

The above action is nothing but the $\left(C^{3}+A\right)$ WZW action. We have thus showed that:

The super Poisson-Lie duality relates the $\left(C^{3}+A\right)$ WZW model to a sigma model defined on the Lie supergroup $\left(C^{3}+A\right)$ when the dual Lie supergroup is $C^{3} \oplus A_{1,1_{1}}$. . . 
Let us now turn into the dual sigma model. We parametrize the corresponding Lie supergroup $C^{3} \oplus A_{1,1} . i$ with bosonic coordinates $\{\tilde{x}, \tilde{y}\}$ and fermionic ones $\{\tilde{\psi}, \tilde{\chi}\}$ so that its elements can be written in the same parametrization (3.5) as:

$$
\tilde{g}=e^{\tilde{\chi} \tilde{Q}}-e^{\tilde{y} \tilde{H}+\tilde{x} \tilde{Z}} e^{\tilde{\psi} \tilde{Q}+}
$$

from which we can read off the $\tilde{R}_{+i}^{(l)}$ 's as

$$
\tilde{R}_{ \pm_{\tilde{H}}}^{(l)}=\partial_{ \pm} \tilde{y}, \quad \tilde{R}_{ \pm_{\tilde{Z}}}^{(l)}=\partial_{ \pm} \tilde{x}, \quad \tilde{R}_{ \pm_{\tilde{Q}_{+}}}^{(l)}=\partial_{ \pm} \tilde{\psi}, \quad \tilde{R}_{ \pm_{\tilde{Q}_{-}}}^{(l)}=-\partial_{ \pm} \tilde{\psi} \frac{\tilde{x}}{2}+\partial_{ \pm} \tilde{\chi}
$$

and then using Eqs. (2.10) for the dual model we obtain

$$
\tilde{\Pi}_{i j}(\tilde{g})=\left(\begin{array}{cccc}
0 & 0 & 0 & -\tilde{\psi} \\
0 & 0 & 0 & 0 \\
0 & 0 & 0 & 0 \\
\tilde{\psi} & 0 & 0 & -\tilde{x}
\end{array}\right)
$$

Finally, by substituting the above relation in the second equation of (2.8) the dual model action is obtained as follows:

$$
\begin{gathered}
\tilde{S}=\frac{1}{2} \int d \xi^{+} d \xi^{-}\left(\partial_{+} \tilde{y} \partial_{-} \tilde{x}+\partial_{+} \tilde{x} \partial_{-} \tilde{y}+\partial_{+} \tilde{x} \tilde{\psi} \partial_{-} \tilde{\psi}+\partial_{+} \tilde{\psi} \tilde{\psi} \partial_{-} \tilde{x}\right. \\
\left.-\partial_{+} \tilde{\psi} \tilde{x} \partial_{-} \tilde{\psi}-\partial_{+} \tilde{\psi} \partial_{-} \tilde{\chi}+\partial_{+} \tilde{\chi} \partial_{-} \tilde{\psi}\right) .
\end{gathered}
$$

The above action can be rewritten as the following one up to total derivatives

$$
\tilde{S}=\frac{1}{2} \int d \xi^{+} d \xi^{-}\left(\partial_{+} \tilde{y} \partial_{-} \tilde{x}+\partial_{+} \tilde{x} \partial_{-} \tilde{y}-\partial_{+} \tilde{\psi} \partial_{-} \tilde{\chi}+\partial_{+} \tilde{\chi} \partial_{-} \tilde{\psi}-\partial_{+} \tilde{\psi} 3 \tilde{x} \partial_{-} \tilde{\psi}\right) .
$$

Comparing the above action with the sigma model action of the form (2.1), the metric $\tilde{G}_{\Upsilon \Lambda}$ and the tensor field $\tilde{B}_{\Upsilon \Lambda}$ take the following forms

$$
\tilde{G}_{\Upsilon \Lambda}=\left(\begin{array}{cccc}
0 & 1 & 0 & 0 \\
1 & 0 & 0 & 0 \\
0 & 0 & 0 & 1 \\
0 & 0 & -1 & 0
\end{array}\right), \quad \tilde{B}_{\Upsilon \Lambda}=\left(\begin{array}{cccc}
0 & 0 & 0 & 0 \\
0 & 0 & 0 & 0 \\
0 & 0 & 3 \tilde{x} & 0 \\
0 & 0 & 0 & 0
\end{array}\right) .
$$

Note that the background matrix of the dual model depends only on the bosonic field $\tilde{x}$. We see that in this example, the super Poisson-Lie T-duality transforms the role of the fermionic field $\chi$ in the model (4.6) to the bosonic field $\tilde{x}$ on the dual model (4.11). Furthermore we observe that the metric is Ricci flat, i.e., $R=R_{\Upsilon \Lambda}=0$. As a WZW model, the original model should be conformally invariant, but to prove the conformal invariance of the dual model we look at the one-loop $\beta$-function equations. Taking into account the dilaton field $\varphi$ for the sigma model (2.1), the one-loop $\beta$-function equations [16] are given by

$$
\begin{aligned}
& \beta_{\Upsilon \Lambda}^{(G)}=R_{\Upsilon \Lambda}+\frac{1}{4} H_{\Upsilon \Delta \Xi} H_{\Lambda}^{\Xi \Delta}+2 \vec{\nabla}_{\Upsilon} \vec{\nabla}_{\Lambda} \varphi=0, \\
& \beta_{\Lambda \Delta}^{(B)}=(-1)^{\Upsilon} \vec{\nabla}^{\Upsilon}\left(e^{-2 \varphi} H_{\Upsilon \Lambda \Delta}\right)=0,
\end{aligned}
$$




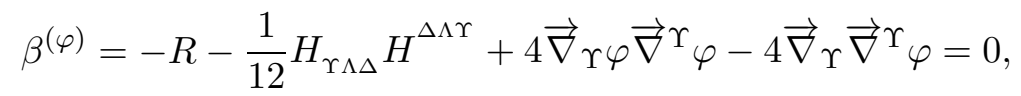

where

$$
H_{\Upsilon \Lambda \Delta}=B_{\Upsilon \Lambda} \frac{\overleftarrow{\partial}}{\partial \Phi^{\Delta}}+(-1)^{\Upsilon(\Lambda+\Delta)} B_{\Lambda \Delta} \frac{\overleftarrow{\partial}}{\partial \Phi^{\Upsilon}}+(-1)^{\Delta(\Upsilon+\Lambda)} B_{\Delta \Upsilon} \frac{\overleftarrow{\partial}}{\partial \Phi^{\Lambda}}
$$

is the torsion field. We note that Eqs. (4.13) are equations of motion of the following effective action on supermanifold [16]

$$
S_{\text {eff }}=\int d^{m, n} \Phi \sqrt{G} e^{-2 \varphi}\left(R+4 \vec{\nabla}_{\Upsilon \varphi} \vec{\nabla}^{\Upsilon} \varphi+\frac{1}{12} H_{\Upsilon \Lambda \Delta} H^{\Delta \Lambda \Upsilon}\right) .
$$

After some calculations, one can find that the dilaton for the dual model is constant and the only non-zero component of $H$ is $H_{233}=3$. Putting these in (4.13), one verifies that those equations are satisfied. It is straightforward to verify that $H_{\Upsilon \Lambda \Delta} H^{\Delta \Lambda \Upsilon}=0$, and thus the effective action is vanished. Therefore, one can coclude that the dual model is conformal invariant for all loops. In this way, a pair of conformal sigma models related by super Poisson-Lie T-duality is constructed by starting with the $\left(\left(C^{3}+A\right), C^{3} \oplus A_{1,1} \cdot i\right)$ Drinfel'd superdouble. Note that the original model (WZW model on the $\left(C^{3}+A\right)$ ) has also $R=R_{\Upsilon \Lambda}=0, H_{144}=-1$ and $H_{\Upsilon \Lambda \Delta} H^{\Delta \Lambda \Upsilon}=0$, such that the effective action for the original model is also vanished. But this is not the whole story. Indeed, one can show that the dual action (4.11) is the action of the WZW model on the Lie supergroup $\left(C^{3}+A\right) \cdot i$ where its Lie superalgebra has the following non-zero (anti)commutation relations:

$$
\left[\hat{Z}, \hat{Q_{+}}\right]=\hat{Q_{-}}, \quad\left\{\hat{Q_{+}}, \hat{Q_{+}}\right\}=-\hat{H} .
$$

Note that to construct the WZW model (4.11) on the Lie supergroup $\left(C^{3}+A\right) \cdot i$ we must choose the following convenient parametrization

$$
\hat{g}=e^{\left(\frac{\hat{\chi}}{3}+\frac{\hat{x} \hat{\psi}}{2}\right)} \hat{Q_{-}} e^{\frac{-\hat{y}}{3} \hat{H}-\hat{x} \hat{Z}} e^{\hat{\psi} \hat{Q_{+}}} .
$$

Thus, in this way, the super Poisson-Lie T-duality relates the WZW models to each other.

\section{Preservation of the $\mathrm{N}=(2,2)$ structure under super Poisson-Lie $\mathrm{T}$ - duality}

We know that the extended superconformal (especially $\mathrm{N}=2$ ) current algebras are equivalent to the Manin triples [23]. The algebraic structure of the $\mathrm{N}=(2,2)$ supersymmetric WZW model is equivalent to the Manin triple (or Lie bialgebra) structure 24]. Furthermore, the study of the $\mathrm{N}=(2,2)$ structure under the Poisson-Lie T-duality is an interesting problem (see 25]). Here in this paper according to the above calculations, the super Poisson-Lie T-duality relates the WZW model on the Lie supergroup $\left(C^{3}+A\right)$ to the other WZW model on the Lie supergroup $\left(C^{3}+A\right) \cdot i$. Thus, to construct the $\mathrm{N}=(2,2) \mathrm{WZW}$ model on the Lie supergroup $\left(C^{3}+A\right)$, according to $(3.9)$ we have

$$
S_{N=(2,2)}(g)=\int d \xi^{+} d \xi^{-} d^{2} \theta(-1)^{\Upsilon} D_{+} \Phi^{\Upsilon}\left(G_{\Upsilon \Lambda}(\Phi)+B_{\Upsilon \Lambda}(\Phi)\right) D_{-} \Phi^{\Lambda},
$$


in which the superfields $\Phi^{\Upsilon}$ include all the bosonic coordinates $(y, x)$ and the fermionic ones $(\psi, \chi)$ (parameters of the Lie supergroup $\left(C^{3}+A\right)$ ) where they are functions of the worldsheet coordinates $(\tau, \sigma)$ and Grassmannian ones $\left(\theta_{1}, \theta_{2}\right)^{5}$, and the background matrix is also given by (3.10). By composing the invariance of the above action under the supersymmetry transformations

$$
\delta_{\epsilon}^{1} \Phi^{\Upsilon}=i\left(\epsilon^{+} Q_{+}+\epsilon^{-} Q_{-}\right) \Phi^{\Upsilon}
$$

and

$$
\delta_{\epsilon}^{2} \Phi^{\Upsilon}=\epsilon^{+} D_{+} \Phi^{\Lambda} J_{+}{ }^{\Upsilon}{ }_{\Lambda}(\Phi)+\epsilon^{-} D_{-} \Phi^{\Lambda} J_{-}{ }^{\Upsilon}{ }_{\Lambda}(\Phi),
$$

where $Q_{ \pm}$and $D_{ \pm}$are supersymmetry generators and superderivatives, respectively, the $\epsilon^{ \pm}$are the supersymmetry parameters and $J_{ \pm \Lambda}^{\Upsilon} \in T G \otimes T^{*} G$, one can find that the $J_{ \pm \Lambda}^{\Upsilon}$ must be complex structure on Lie supergroup $G$ where its generalized covariant derivative with respect to extended Christoffel symbols $\left(\Gamma_{\Lambda \Xi}^{ \pm \Upsilon}=\Gamma_{\Lambda \Xi}^{\Upsilon} \pm(-1)^{\Omega} G^{\Upsilon \Omega} H_{\Omega \Lambda \Xi}\right)$ must be zero [26], i.e., the background supermanifold $G$ must have super bi-Hermitian structure. If we use the non-coordinate basis for Lie supergroup $G$ then we obtain the form of the algebraic super bi-Hermitian structure as in the bosonic case [25], [27].

We note that the $\left(\mathcal{C}^{3}+\mathcal{A}\right)$ Lie superalgebra is a Drinfel'd super double as $\mathcal{D}=$ $\left(\left(\mathcal{A}_{1,1}+\mathcal{A}\right), \mathcal{I}_{(1 \mid 1)}\right)$, so those equations automatically satisfy for the model (5.1). Hence, the Lie supergroup $\left(C^{3}+A\right)$ has a super bi-Hermitian structure or we have a $\mathrm{N}=(2,2)$ structure on the model (5.1). On the other hand, the dual model is also equivalent to the $\mathrm{N}=(2,2)$ WZW model on the Lie supergroup $\left(C^{3}+A\right) \cdot i$, because the $\left(\mathcal{C}^{3}+\mathcal{A}\right) \cdot i$ Lie superalgebra is also a Drinfel'd superdouble. In this way, the $\mathrm{N}=(2,2)$ structure is preserved under the super Poisson-Lie T-duality. Note that this is the first example of this type.

\section{Conclusion and remarks}

We showed that WZW model on the Lie supergroup $\left(C^{3}+A\right)$ contains super Poisson-Lie symmetry when the dual Lie supergroup is $C^{3} \oplus A_{1,1} . i$. Then, we constructed the mutually

T-dual sigma models on the Drinfel'd superdouble $\left(\left(C^{3}+A\right), C^{3} \oplus A_{1,1} \cdot i\right)$, and showed that the dual model is also conformal invariant. It is quite interesting that the dual model is equivalent to the WZW model on the Lie supergroup $\left(C^{3}+A\right)$. $i$. Furthermore, using the fact that the $\left(\mathcal{C}^{3}+\mathcal{A}\right) \cdot i$ Lie superalgebra is a Drinfel'd superdouble we saw that the $\mathrm{N}=(2,2)$ structure is preserved under the super Poisson-Lie T-duality. Of course, there are some open problems related to our results. As an example, one can see that WZW model on the Lie supergroup $\left(C^{3}+A\right) \cdot i$ (the dual model) has also super Poisson-Lie symmetry with the dual Lie supergroup $\left(2 A_{1,1}+2 A\right) . i$ [21], and it seems this process can be continued. In this way, we have a hierarchy of WZW models related by the super Poisson-Lie T-duality; such that it is different from plurality, because the doubles are non-isomorphic. For exact discussion, one must first obtain and classify all Lie superbialgebras of $\left(\mathcal{C}^{3}+\mathcal{A}\right)$, but this needs another challenge. Some of these problems are under investigation.

\footnotetext{
${ }^{5}$ Note that the action (5.1) has been written in the $\mathrm{N}=1$ formalism (see [24]).
} 
Acknowledgments: This research was supported by a research fund No. 401.231 from Azarbaijan Shahid Madani university.

\section{References}

[1] V. Schomerus and H. Saleur, Nucl. Phys. B 734 (2006) 221-245 hep-th/0510032].

[2] G.Gotz, T.Quella and V.Schomerus, J. High Energy Phys. 03 (2007) 003 [hep-th/0610070].

[3] H. Saleur and V. Schomerus, Nucl. Phys. B 775 (2007) 312-340 hep-th/0611147].

[4] M. Henneaux and L. Mezincescu, Phys. Lett. B 152 (1985) 340-342.

[5] R. R. Metsaev and A. A. Tseytlin, Nucl. Phys. B 533 (1998) 109 hep-th/9805028.

[6] N.Berkovits, C.Vafa and E.Witten, J. High Energy Phys. 03 (1999) 018 hep-th/9902098].

[7] N. Berkovits, M. Bershadsky, T. Hauer, S. Zhukov and B. Zwiebach, Nucl. Phys. B 567 (2000) 61-86 hep-th/9907200.

[8] T. H. Buscher, Phys. Lett. B 201 (1988) 466-472.

[9] M. Rocek and E. Verlinde, Nucl. Phys. B 373 (1992) 630.

[10] E. Kiritsis, Nucl. Phys. B 405 (1993) 109; Mod. Phys. Lett. A 6 (1991) 2871; S. F. Hassan and A. Sen, Nucl. Phys. B 405 (1993) 143.

[11] X. C. de la Ossa and F. Quevedo, Nucl. Phys. B 403 (1993) 377

[12] A. Givoen and M. Rocek, Nucl. Phys. B 421 (1994) 173; E. Tyurin, Phys. Lett. B 348 (1995) 386; M. Gasperini, R. Ricci and G. Veneziano, Phys. Lett. B 319 (1993) 438; S. Elitzur, A.Giveon, E. Rabinovici, A. Schwimmer and G. Veneziano, Nucl. Phys. B 435 (1995) 147; E. Alvarez, A. Alvarez-Gaume and Y. Lozano, Nucl. Phys. B 424 (1994) 155; N. Mohammedi, Phys. Lett. B 414 (1997) 104 hep-th/9709071].

[13] C. Klimčik and P. Ševera, Phys. Lett. B 351 (1995) 455 hep-th/9502122]; Phys. Lett. B 372 (1996) 65 [hep-th/9512040]; C. Klimcik, Nucl. Phys. 46 (Proc. Suppl.) (1996) 116 hep-th/9509095.

[14] A. Alekseev, C. Klimčik and A. Tseytlin, Nucl. Phys. B 458 (1996) 530 hep-th/9509123.

[15] A. Eghbali and A. Rezaei-Aghdam, J. High Energy Phys. 09 (2009) 094 arXiv:0901.1592.

[16] A. Eghbali and A. Rezaei-Aghdam, J. High Energy Phys. 01 (2012) 151 arXiv:1107.2041.

[17] A. Eghbali and A. Rezaei-Aghdam, Nucl. Phys. B 866 (2013) 26-42 [arXiv:1207.2304].

[18] B. DeWitt, Supermanifolds, Cambridge University Press 1992.

[19] A. Eghbali, A. Rezaei-Aghdam and F. Heidarpour, J. Math. Phys. 51 (2010) 103503 arXiv:0911.1760.

[20] N. Backhouse, J. Math. Phys. 19 (1978) 2400-2402.

[21] A. Eghbali and A. Rezaei-Aghdam, J. Geom. Phys. 65 (2013) 7 25 [arXiv:1112.0652].

[22] A. Eghbali, A. Rezaei-Aghdam and F. Heidarpour, J. Math. Phys. 51 (2010) 073503 arXiv:0901.4471.

[23] S. Parkhomenko, Sov. Phys. JETP 75 (1992) 1 - 3.

[24] Ulf Lindstrom and Maxim Zabzine, Phys. Lett. B 560 (2003) 108-115 hep-th/0212042.

[25] M. Ebrahimi and A. Rezaei-Aghdam, arXiv:1204.5895].

[26] S. J. Gate, C. M. Hull and M. Roček, Nucl. Phys. B 248 (1984) 157.

[27] A. Rezaei-Aghdam and M. Sephid, J. Phys. A 43 (2010) 325210 arXiv:1002.4285. 